Research Notes

\title{
A Note on a New Method of Investigation of Solutions of Boundary Value Problems for the Elliptic Type Equations of the First Order
}

\author{
${ }^{2}$ Nihan A. Aliyev, ${ }^{1}$ Alexander A. Katz and ${ }^{2}$ Metanet B. Mursalova \\ ${ }^{1}$ Department of Mathematics and Computer Science, \\ Saint John's College of LAS, Saint John's University, NY, United States \\ ${ }^{2}$ Department of Mathematics, Baku State University, Azerbaijan
}

\author{
Article history \\ Received: 29-10-2017 \\ Revised: 20-12-2017 \\ Accepted: 09-01-2018 \\ Corresponding Author: \\ Alexander A. Katz \\ Department of Mathematics and \\ Computer Science, Saint John's \\ College of LAS, Saint John's \\ University, NY, United States \\ E-mail:katza@stjohns.edu
}

\begin{abstract}
The paper deals with investigation of the solutions of the boundary value problem for the Cauchy-Riemann equation on the curvelinear strip in the real plane. Here the boundary conditions are in the special type and are fundamental with respect to the direction solutions are used. An analytic form of the solution of considered boundary value problem is obtained.
\end{abstract}

Keywords: Cauchy-Riemann Equation, Solutions which are Fundamental with Respect to Direction, Main Relation, Necessary Condition, Solution of the Boundary Problem

\section{Introduction}

As is known, in the boundary value problem for an ordinary differential equation the number of boundary conditions coinsides with the order of the differential equation. Unlike boundary value problem for a linear ordinary differential equations, in the boundary value problem for partial differential equations of the elliptic type the number of local boundary conditions coinsides with the half of the order of equation under consideration (Bitsadze and Samarskii, 1969). The canonic form of the equations of elliptic type is the Laplas equation for which the conditions of Dirichlet, Neumann, Poincare, the 3rd boundary value problem and etc., were considered (Babich et al., 1964).

As the Cauchy-Riemann equation is the partial differential equation of the first order of the eliptic type, then the local boundary conditions mentioned above are unacceptable and the Dirichlet problem for the CauchyRiemann equation that was considered by some authors is incorrect (Begehr, 2005).

Different boundary value problems with the non-local conditions for the Cauchy-Riemann equation were considered in (Aliyev et al., 2010; Aliev and Mamedov, 1983; Aliev and Mechtiyev, 2002; Aliyev and Mursalova, 2016; Mekhtiyev et al., 2009).

Non-local boundary problem with conditions describing the relationship between the values of the unknown function on the part of the boundary and its values on some line lying within the considered domain was studied in (Bitsadze and Samarskii, 1969).
In the presented paper non-local boundary conditions relate values of the unknown function on the part of boundary with the values of this function on the line lying outside the considered domain.

\section{Statement of the Problem}

$$
\text { Let } D=\left\{x=\left(x_{1}, x_{2}\right): x_{1} \in \mathbb{R}, x_{2} \in\left(0, \gamma_{2}\left(x_{1}\right)\right), \gamma_{2}\left(x_{1}\right)>\right.
$$
$0\} \subset \mathbb{R}^{2}$ is curvelinear strip in the real plane $\mathbb{R}^{2}$. Let us consider the following problem:

$l u \equiv \frac{\partial u(x)}{\partial x_{2}}+i \frac{\partial u(x)}{\partial x_{1}}=0, x \in D \subset \mathbb{R}^{2}$

$u\left(x_{1}, \gamma_{2}\left(x_{1}\right)\right)=\alpha\left(x_{1}\right) u\left(x_{1}-i \gamma_{2}\left(x_{1}\right), 0\right)+\varphi\left(x_{1}\right), x_{1} \in \mathbb{R}$,

here, $\quad i=\sqrt{-1}, \alpha\left(x_{1}\right)$ and $\varphi\left(x_{1}\right)$ are given continuous functions of $x_{1} \in \mathbb{R}^{2}$.

\section{Fundamental Solutions}

As it is known (Vladimirov, 1988), the function:

$$
U(x-\xi)=\frac{1}{2 \pi} \cdot \frac{1}{x_{2}-\xi_{2}+i\left(x_{1}-\xi_{1}\right)}
$$

is the fundamental solution of the Cauchy-Riemann Equation (1), which may be obtained by using the Fourier transform. As it was noted in (Bitsadze, 1968), the 
difference between two known fundamental solutions of the same equation is the regular solution of the corresponding homogeneous equation. Let us now consider solutions of Equation (1), that are fundamental with respect to direction. It is easy to see that the function:

$$
U_{2}(x-\xi)=\theta\left(x_{2}-\xi_{2}\right) \delta\left(x_{1}-\xi_{1}-i\left(x_{2}-\xi_{2}\right)\right)
$$

is the fundamental solution of Equation (1) with respect to direction $x_{2}$ and function:

$$
U_{1}(x-\xi)=-i \theta\left(x_{1}-\xi_{1}\right) \delta\left(x_{2}-\xi_{2}+i\left(x_{1}-\xi_{1}\right)\right)
$$

is the fundamental solution of Equation (1) with respect to direction $x_{1}$. Here $\theta(x)$ is the unital Heaviside function and $\delta(x-\xi)$ is the Dirac delta function with the complex argument. Indeed:

$$
\begin{aligned}
& \frac{\partial U_{2}(x-\xi)}{\partial x_{2}}=\delta\left(x_{2}-\xi_{2}\right) \cdot \delta\left(x_{1}-\xi_{1}-i\left(x_{2}-\xi_{2}\right)\right)-i \theta\left(x_{2}-\xi_{2}\right) . \\
& \delta^{\prime}\left(x_{1}-\xi_{1}-i\left(x_{2}-\xi_{2}\right)\right)=\delta(x-\xi)-i \theta\left(x_{2}-\xi_{2}\right) . \\
& \cdot \delta^{\prime}\left(x_{1}-\xi_{1}-i\left(x_{2}-\xi_{2}\right)\right), \\
& \frac{\partial U_{2}(x-\xi)}{\partial x_{1}}=\theta\left(x_{2}-\xi_{2}\right) \cdot \delta^{\prime}\left(x_{1}-\xi_{1}-i\left(x_{2}-\xi_{2}\right)\right) .
\end{aligned}
$$

Then:

$$
\frac{\partial U_{2}(x-\xi)}{\partial x_{2}}+i \frac{\partial U_{2}(x-\xi)}{\partial x_{1}}=\delta(x-\xi)
$$

By analogy:

$$
\frac{\partial U_{1}(x-\xi)}{\partial x_{2}}+i \frac{\partial U_{1}(x-\xi)}{\partial x_{1}}=\delta(x-\xi) .
$$

But their difference:

$$
U_{2}(x-\xi)-U_{1}(x-\xi)=U_{0}(x-\xi)
$$

is not a regular solution of the Cauchy-Riemann Equation (1), althrough it is a solution of this equation.

\section{Main Relation}

We prove the following.

\section{Theorem 1.}

Let $\gamma_{2}\left(x_{1}\right)>0$ be continuously differentiable function for $x_{1} \in \mathbb{R}$. If:

$$
\begin{aligned}
& \lim _{x_{1} \rightarrow \infty} \int_{0}^{\gamma_{2}\left(x_{1}\right)} \theta\left(x_{2}-\xi_{2}\right) \delta\left(x_{1}-\xi_{1}-i\left(x_{2}-\xi_{2}\right)\right) u(x) d x \\
& =\lim _{x_{1} \rightarrow-\infty} \int_{0}^{\gamma_{2}\left(x_{1}\right)} \theta\left(x_{2}-\xi_{2}\right) \delta\left(x_{1}-\xi_{1}-i\left(x_{2}-\xi_{2}\right)\right) u(x) d x_{2}
\end{aligned}
$$

Then for each analytic function defined in $D \subset \mathbb{R}^{2}$, the following main relation is valid:

$$
\begin{aligned}
& -\int_{R} \theta\left(-\xi_{2}\right) \delta\left(x_{1}-\xi_{1}+i \xi_{2}\right) u\left(x_{1}, 0\right) d x_{1} \\
& +\int_{R} \theta\left(\gamma_{2}\left(x_{1}\right)-\xi_{2}\right) \delta\left(x_{1}-\xi-i\left(\gamma_{2}\left(x_{1}\right)-\xi_{2}\right)\right)\left[1-\gamma_{2}^{\prime}\left(x_{1}\right)\right] \\
& u\left(x_{1}, \gamma_{2}\left(x_{1}\right)\right) d x_{1}=\left\{\begin{array}{c}
u(\xi), \xi \in D \subset R_{2} \\
\frac{1}{2} u(\xi), \xi \in \partial D \subset R_{2}
\end{array} .\right.
\end{aligned}
$$

\section{Proof}

Multiplying (1) on fundamental solution (4), integrating by domain $D$ and applying the OstroqradskyGauss formula we get:

$$
\begin{aligned}
& O=\int_{D} \frac{\partial u(x)}{\partial x_{2}} U_{2}(x-\xi) d x+i \int_{D} \frac{\partial u(x)}{\partial x_{1}} U_{2}(x-\xi) d x \\
& =\int_{\partial D} u(x) U_{2}(x-\xi)\left[\cos \left(v, x_{2}\right)+i \cos \left(v, x_{1}\right)\right] d x \\
& -\int_{D} u(x)\left[\frac{\partial U_{2}(x-\xi)}{\partial x_{2}}+i \frac{\partial U_{2}(x-\xi)}{\partial x_{1}}\right] d x
\end{aligned}
$$

here $v$ is an external normal to the boundary $\partial D$ of the domain $D$. Taking into account (6) and properties of the Dirac $\delta$-function (Vladimirov, 1988), we obtain from (10) following main relation:

$$
\begin{aligned}
& -\int_{R} \theta\left(-\xi_{2}\right) \delta\left(x_{1}-\xi_{1}+i \xi_{2}\right) u\left(x_{1}, 0\right) d x_{1} \\
& +\int_{R} \theta\left(\gamma_{2}\left(x_{1}\right)-\xi_{2}\right) \delta\left(x_{1}-\xi_{1}-i\left(\gamma_{2}\left(x_{1}\right)-\xi_{2}\right)\right) \\
& {\left[1-\gamma_{2}^{\prime}\left(x_{1}\right)\right] u\left(x_{1}, \gamma_{2}\left(x_{1}\right)\right) d x_{1}} \\
& = \begin{cases}u(\xi), \quad \xi \in D \subset R^{2} \\
\frac{1}{2} u(\xi), & \xi \in \partial D \subset R^{2}\end{cases}
\end{aligned}
$$

\section{Remark 1}

In the main relation (9) the first correlation is representation of an arbitrary analytic function when $\xi \in$ $D$ and the second is the necessary condition.

\section{Necessary Conditions}

From the main relation (9) we get:

$$
\left\{\begin{array}{c}
u\left(\xi_{1}, 0\right)=-u\left(\xi_{1}, 0\right)+2 \int_{R} \delta\left(x_{1}-\xi_{1}-i \gamma_{2}\left(\xi_{1}\right)\right) u\left(x_{1}, \gamma_{2}\left(x_{1}\right)\right) \\
\cdot\left[1-\gamma_{2}^{\prime}\left(x_{1}\right)\right] d x_{1} \\
u\left(\xi_{1}, \gamma_{2}\left(\xi_{1}\right)\right)=2 \int_{R} \theta\left(\gamma_{2}\left(x_{1}\right)-\gamma_{2}\left(\xi_{1}\right)\right) . \\
\cdot \delta\left(x_{1}-\xi_{1}-i\left(\gamma_{2}\left(x_{1}\right)-\gamma_{2}\left(x_{1}\right)\right)\right) u\left(x_{1}, \gamma_{2}\left(x_{2}\right)\right)\left[1-i \gamma_{2}^{\prime}\left(x_{1}\right)\right] d x_{1}
\end{array}\right.
$$


or:

$$
\begin{aligned}
& u\left(\xi_{1}, 0\right) \\
& =\int_{R} u\left(x_{1}, \gamma_{2}\left(x_{1}\right)\right) \delta\left(x_{1}-\xi_{1}-i \gamma_{2}\left(x_{1}\right)\right)\left[1-\gamma_{2}^{\prime}\left(x_{1}\right)\right] d x_{1} \\
& u\left(\xi_{1}, \gamma_{2}\left(\xi_{1}\right)\right)=u\left(\xi_{1} \gamma_{2}\left(\xi_{1}\right)\right)
\end{aligned}
$$

As we see, here the second necessary condition turns into identity, so we get just one necessary condition $\left(11^{1}\right)$

\section{Remark 2.Note 2}

Using fundamental solution of the Cauchy-Riemann equation, which was obtained in (Vladimirov, 1988) we will obtain two necessary conditions containing singular part (Aliyev and Mursalova, 2016).

Consider now the following equation with respect to $x_{1}$ :

$x_{1}-\xi_{1}-i \gamma_{2}\left(x_{1}\right)=0$

Let its solution is in the form:

$x_{1}=\xi_{1}+i \sigma_{2}\left(\xi_{1}\right)$

here $\sigma_{2}\left(\xi_{1}\right)$ is while unknown function.

Then we get:

$$
\xi_{1}+i \sigma_{2}\left(\xi_{1}\right)-\xi_{1}-i \gamma_{2}\left(\xi_{1}+i \sigma_{2}\left(\xi_{1}\right)\right) \equiv 0
$$

and:

$$
x_{1} \equiv x_{1}-i \gamma_{2}\left(x_{1}\right)+i \sigma_{2}\left(x_{1}-i \gamma_{2}\left(x_{1}\right)\right)
$$

or:

$$
\sigma_{2}\left(\xi_{1}\right) \equiv \gamma_{2}\left(\xi_{1}+i \sigma_{2}\left(\xi_{1}\right)\right)
$$

and

$$
\gamma_{2}\left(x_{1}\right) \equiv \sigma_{2}\left(x_{1}-i \gamma_{2}\left(x_{1}\right)\right)
$$

and we obtained the necessary condition $\left(11^{1}\right)$ in form:

$$
u\left(\xi_{1}, 0\right)=u\left(\xi_{1}+i \sigma_{2}\left(\xi_{1}\right), \gamma_{2}\left(\xi_{1}+i \sigma_{2}\left(\xi_{1}\right)\right)\right)
$$

or, taking into account (14):

$$
u\left(\xi_{1}, 0\right)=u\left(\xi_{1}+i \sigma_{2}\left(\xi_{1}\right), \sigma_{2}\left(\xi_{1}\right)\right) .
$$

From the (12) and (13) we get:

$$
\sigma_{2}\left(\xi_{1}\right)=-i\left(x_{1}-\xi_{1}\right)=-i \cdot i \gamma_{2}\left(x_{1}\right)=\gamma_{2}\left(x_{1}\right)
$$

and from (13), (16), (17) we get:

$$
u\left(\xi_{1}, 0\right)=u\left(x_{1}, \gamma_{2}\left(x_{1}\right)\right)
$$

Taking into account (12) in the last expression we get:

$$
u\left(x_{1}, \gamma_{2}\left(x_{1}\right)\right)=u\left(x_{1}-i \gamma_{2}\left(x_{1}\right), 0\right) \text {. }
$$

\section{Solution of the Boundary Value Problem}

(1), (2)

Let us assume now that:

$\alpha\left(x_{1}\right) \neq 1$,

$x_{1} \in \mathbb{R}$.

The following theorem is valid.

\section{Theorem 2.}

Let the conditions of Theorem 1 are satisfied. Under condition (20), there exists a unique solution of problem (1), (2), representable in the form:

$u\left(\xi_{1}, \xi_{2}\right)=\frac{\varphi\left(\xi_{1}-i \xi_{2}+i \sigma_{2}\left(\xi_{1}-i \xi_{2}\right)\right)}{1-\alpha\left(\xi_{1}-i \xi_{2}+i \sigma_{2}\left(\xi_{1}-i \xi_{2}\right)\right)}$.

\section{Proof}

Indeed, going back to the boundary condition (2) and using the necessary condition (11) we obtain:

$$
u\left(x_{1}, \gamma_{2}\left(x_{1}\right)\right)=\alpha\left(x_{1}\right) u\left(x_{1}, \gamma_{2}\left(x_{1}\right)\right)+\varphi\left(x_{1}\right)
$$

or:

$$
u\left(x_{1}, \gamma_{2}\left(x_{1}\right)\right)=\frac{\varphi\left(x_{1}\right)}{1-\alpha\left(x_{1}\right)}, x_{1} \in \mathbb{R} .
$$

Then the solution of the problem (1), (2) may be obtained from (9) in following form:

$$
\begin{aligned}
& u(\xi)=\int_{\mathbb{R}} \frac{\varphi\left(x_{1}\right)}{1-\alpha\left(x_{1}\right)} \theta\left(\gamma_{2}\left(x_{1}\right)-\xi_{2}\right) \\
& \delta\left(x_{1}-\xi_{1}-i\left(\gamma_{2}\left(x_{1}\right)-\xi_{2}\right)\right)\left(1-\gamma_{2}^{\prime}\left(x_{1}\right)\right) d x_{1}
\end{aligned}
$$

or:

$$
\begin{aligned}
& u\left(\xi_{1}, \xi_{2}\right)=\frac{\varphi\left(\xi_{1}-i \xi_{2}+i \sigma_{2}\left(\xi_{1}-i \xi_{2}\right)\right)}{1-\alpha\left(\xi_{1}-i \xi_{2}+i \sigma_{2}\left(\xi_{1}-i \xi_{2}\right)\right)} . \\
& \theta\left(\gamma_{2}\left(\xi_{1}-i \xi_{2}+i \sigma_{2}\left(\xi_{1}-i \xi_{2}\right)\right)\right) .
\end{aligned}
$$




$$
u\left(\xi_{1}, \xi_{2}\right)=\frac{\varphi\left(\xi_{1}-i \xi_{2}+i \sigma_{2}\left(\xi_{1}-i \xi_{2}\right)\right)}{1-\alpha\left(\xi_{1}-i \xi_{2}+i \sigma_{2}\left(\xi_{1}-i \xi_{2}\right)\right)} .
$$

It is obvious that function (21) satisfies Equation (1). Let us show that it satisfies condition (2) as well:

$$
\begin{aligned}
& u\left(\xi_{1}, \gamma_{2}\left(\xi_{1}\right)\right)-\alpha\left(\xi_{1}\right) u\left(\xi_{1}-i \gamma_{2}\left(\xi_{1}\right), 0\right) \stackrel{(17)}{=} u\left(\xi_{1}, \gamma_{2}\left(\xi_{1}\right)\right) \\
& -\alpha\left(\xi_{1}\right) u\left(\xi_{1}, \gamma_{2}\left(\xi_{1}\right)\right)=u\left(\xi_{1}, \gamma_{2}\left(\xi_{1}\right)\right)\left[1-\alpha\left(\xi_{1}\right)\right]_{(22)}^{=} \varphi\left(\xi_{1}\right)
\end{aligned}
$$

\section{Acknowledgement}

Authors are thankful to the referee for helpful comments.

\section{Author's Contributions}

All authors equally contributed in this work.

\section{Ethics}

This article is original and contains unpublished material. The corresponding author confirms that all of the other authors have read and approved the manuscript and there are no ethical issues involved.

\section{References}

Aliev, N.A. and F.O. Mamedov, 1983. A problem of mixed type for a first-order model equation. (Russian) Izv. Akad. Nauk Azerba 1dzhan. SSR Ser. Fiz.-Tekhn. Mat. Nauk, 4: 110-113.

Aliyev, N.A., V.H. Fatemi and M. Jahaosbahi, 2010. Analytic solution for the Cauchy-Riemann equation with non-local boundary conditions in the first semiquarter. (English) J. Sci. (Kharazmi University), 9: 29-40.
Aliev, N.A. and A.A. Mechtiyev, 2002. Investigation of solution of the boundary value problem for the Cauchy-Riemann equation in the bounded plane domain. (English) Sumgait GU, 2: 30-34.

Aliyev, N.A. and M.B. Mursalova, 2016. Fredholm property of the boundary problem in the curvelinear strip. IW NHA, Abstracts, Baku.

Babich, V.M., M.B. Kapilevich, S.G. Mihlin, G.I. Natanson and P.M. Riz et al., 1964. The Linear Equations of Mathematical Physics. 1st Edn., NAUKA, Moscow, pp: 368.

Begehr, H., 2005. Boundary value problems in complex analysis. I. Bol. Asoc. Mat. Venez., 12: 65-85.

Bitsadze, A.V., 1968. Boundary Value Problems for Second Order Elliptic Equations. 1st Edn., NorthHolland Publishing Co., Amsterdam, pp: 212.

Bitsadze, A.V. and A.A. Samarskii, 1969. Some elementary generalizations of linear elliptic boundary value problems. Dokl. Akad. Nauk SSSR, 185: 739-740.

Mekhtiyev, M.F., N.A. Aliyev and M.R. Fatemi, 2009. On Fredholm property of a boundary value problem for a first order equation with general boundary conditions. (English) Trans. Natl. Acad. Sci. Azerb. Ser. Phys.-Tech. Math. Sci., 29: 221-226.

Vladimirov, V.S., 1988. The Equations of Mathematical Physics. 5th Edn., Moscow, NAUKA, pp: 512. 